\title{
Editorial 2
}

\section{Competency and Referrals in Implant Dentistry}

Implant dentistry is one of the fastest growing treatment modality in dentistry. Implant therapy is a prosthodontic procedure with a surgical component. Placement of dental implants is a procedure, and not considered as a dental specialty. ${ }^{1}$ Despite the ever-growing demand for implant treatments by patients, there is a confusion about what the appropriate training pathway in implant dentistry should be. ${ }^{2}$ Evolving sophisticated diagnostic and therapeutic procedures, such as dental implant therapy, currently taught with different depths and content, result in diverse learning outcomes, not only worldwide but also within dental schools in one region or country. ${ }^{3}$ In addition to the educational variations,

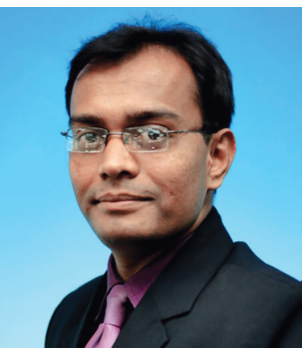
there is also a vast degree of corporate variation in terms of implant companies with variety of implant systems and techniques, protocols, planning softwares and so on. Many implant companies have developed their own educational modules, knowledge delivery methods, workshops and courses. Also the associations and academies including the group of implant therapists have different types of training, examination and casesubmission based programs offering certificates, fellowships and diplomatships. Increased life expectancy and growing demand of implant therapy by the patients advocates the need of formal and basic training at dental undergraduate programs as well. Whether the course they are considering is a university certificate, diploma or Masters level course, or a private certificate course, the programme should have appropriate learning outcomes. ${ }^{2}$ Anyone seeking to embark on implant training to also familiarise themselves with some kind of training standards specifically developed by their country's dental regulatory bodies. ${ }^{4}$ Furthermore, it is important to check the level of mentoring offered to make sure that it is robust and appropriate for their needs and satisfy the clinical aspects of the training. Appropriate end-points of the training programs should also be standardized in terms of university track programs or in terms of examinations.

A general dentist who is trained to place and restore implants may be the appropriate practitioner to provide care for straightforward dental implant procedures. This will vary depending on an individual clinician's amount of training and experience. ${ }^{1}$ However, one should also be aware of the complexity of the implant cases. The practitioner should know when patient should be referred to a specialist (a Prosthodontist, a Periodontist or an Oral and Maxillofacial Surgeon). ${ }^{1}$ Practitioners including specialists should not try to provide care beyond their level of competence and should always be well prepared to refer the patient to appropriate specialist (or seek the referral consultation and/or treatment) in regards to specific procedure in implant therapy. Competence is the blend of knowledge, skills, attitudes and values, appropriate for safe and effective practice by all aspects of the profession. ${ }^{5} \mathrm{~A}$ referral includes determination that assessment, diagnosis, or treatment is required which is beyond the practitioner's competency. An unclear training pathway, inappropriate referrals and a 'hands-off' approach to patients with implants may be putting patients at risk. ${ }^{2}$ The appropriate referral practice beyond one's competence will facilitate the enhanced treatment outcome and patient safety.

\section{References}

1. Agar JR. Dental Implants: Americal College of Prosthodontists (ACP) position statement. 2014:1-3.

2. Barrak F. Taking stock of training in implant dentistry. British Dent J 2016; 221(4):157-158.

3. De Bruyn H, Koole S, Mattheos N, Lang NP. A survey on undergraduate implant dentistry education in Europe. Eur J Dent Educ 2009;13 Suppl 1:3-9.

4. Bartlett D, Brook I M, Ucer C et al. Training standards in implant dentistry. FGDP(UK), 2012.

5. Mattheos N, Ivanovski S, Sambrook P, Klineberg I. Implant dentistry in Australian undergraduate dental curricula: knowledge and competencies for the graduating dentist. Aust Dent J 2010;55(3):333-338.

Pravinkumar G Patil Managing Editor International Journal of Prosthodontics and Restorative Dentistry School of Dentistry, International Medical University Kuala Lumpur, Malaysia 